\title{
Interstellar extinction law near the Galactic equator along the Camelopardalis, Perseus and Cassiopeia border
}

\author{
J. Zdanavičius ${ }^{1}$, V. Straižys ${ }^{1}$, and C. J. Corbally ${ }^{2}$ \\ ${ }^{1}$ Institute of Theoretical Physics and Astronomy, Goštauto 12, Vilnius 2600, Lithuania \\ e-mail: justas@itpa.lt; straizys@itpa.lt \\ 2 Vatican Observatory Research Group, Steward Observatory, Tucson, Arizona 85721, USA \\ e-mail: corbally@as .arizona.edu
}

Received 23 May 2002 / Accepted 19 June 2002

\begin{abstract}
The interstellar reddening law is investigated along the Galactic equator near the Cam, Per and Cas border. We used seven-color photometry of O-B5 stars in the Vilnius photometric system in the optical range, photometry of the ANS orbiting observatory in the ultraviolet range and broad-band color indices $V-K$ in the infrared. In the optical range (345$660 \mathrm{~nm}$ ) the interstellar reddening law is found to be nearly normal. In the ultraviolet wavelengths shorter than the $330 \mathrm{~nm}$ ANS passband, the extinction is found to be slightly larger than the average. Some stars, for example HD 24432, exhibit much stronger ultraviolet extinction which is well seen already in the $345 \mathrm{~nm}$ passband of the Vilnius system. However, such stars are rare. The ratio $R=A_{V} / E_{B-V}$ is found to be 2.9, i.e., it is slightly smaller than the normal. This is confirmed by the study of the wavelength of maximum polarization of reddened stars in the area. Both the ultraviolet anomaly and the smaller $R$ value are in good agreement with the Cardelli et al. (1988) prediction. We conclude that in the investigated area it is safe to use normal ratios of color excesses to calculate reddening-free $Q$-parameters for the classification of stars from photometric data in the optical spectral range.
\end{abstract}

Key words. interstellar medium: extinction - stars: fundamental parameters

\section{Introduction}

A sudden discontinuity of the Milky Way brightness at the Galactic longitudes $>140^{\circ}$ has been well known since the first wide-field surveys of the Galactic system. At this longitude all the tracers of the Perseus spiral arm suddenly disappear (Dodd 1976; Georgelin \& Georgelin 1976; Taylor \& Cordes 1993). The Milky Way reappears only at $\ell=170^{\circ}$, near the Auriga border. This blocking of the light of distant stars is due to the crowding of dust clouds near the Galactic equator which probably belongs to the local Orion spiral arm.

A program of photometric investigation of the Milky Way region at Galactic longitudes $135-155^{\circ}$ (Cassiopeia, Camelopardalis and Perseus) was initiated at the Institute of Theoretical Physics and Astronomy some years ago. The investigation is based on photometry, classification, determination of distances and interstellar extinctions of stars in the Vilnius seven-color photometric system (Table 1). Zdanavičius et al. (1996), hereafter Paper I, have investigated an area of 10 square degrees near the Cas, Per and Cam border at $\ell \approx 143^{\circ}$. Zdanavičius et al. (2001), hereafter Paper II, have investigated an area of similar size near the Cam and Per border at $\ell \approx 149^{\circ}$.

Send offprint requests to: V. Straižys,

e-mail: straizys@itpa.lt
Table 1. Mean wavelengths and half-widths of passbands of the Vilnius photometric system.

\begin{tabular}{lrrrrrrr}
\hline \hline Passband & $U$ & $P$ & $X$ & $Y$ & $Z$ & $V^{*}$ & $S$ \\
\hline & & & & & & & \\
$\lambda_{0}(\mathrm{~nm})$ & 345 & 374 & 405 & 466 & 516 & 544 & 656 \\
$\Delta \lambda(\mathrm{nm})$ & 40 & 26 & 22 & 26 & 21 & 26 & 20 \\
\hline
\end{tabular}

* Medium-band $V$ magnitudes of the Vilnius system have no color equation with respect to $V$ magnitudes of the broad-band $U B V$ system (Straižys 1992). Therefore, we use the same $V$ designation both for medium and broad-band magnitudes.

Zdanavičius \& Zdanavičius (2002), hereafter Paper III, have published a catalog of photometry of 309 stars, mostly of early spectral classes, in a wide area bounded by $2^{\mathrm{h}} 30^{\mathrm{m}}-4^{\mathrm{h}} 20^{\mathrm{m}}$ and $50-63^{\circ}$. For photometric classification of stars in spectral and luminosity classes, we use the interstellar reddening-free $Q$-parameters defined as

$Q_{1234}=\left(m_{1}-m_{2}\right)-\left(E_{12} / E_{34}\right)\left(m_{3}-m_{4}\right)$,

where $m_{1}-m_{2}$ and $m_{3}-m_{4}$ are color indices and $E_{12} / E_{34}$ are ratios of the corresponding color excesses. These ratios are defined by the interstellar reddening law, valid for the dust in front of the area stars. On the other hand, for the transformation of 
color excesses into interstellar extinctions we must know the ratio $R=A / E$ which is defined by the form of the interstellar reddening law in the optical and infrared spectral ranges. Therefore, before applying this method for the classification of stars and their extinction determination, we must know the interstellar extinction law in the area.

In Papers I and II the normal extinction law, as it has been described in the Straižys (1992) monograph, was applied. This law was used both for the calculation of various color excess ratios in the Vilnius system and the ratio $R=A_{V} / E_{Y-V}$ which for early-type stars is equal to 4.16. The normality of the law in these two areas was assumed on the basis of previous investigations of the law in the adjacent areas (Serkowski \& Robertson 1969; Sūdžius 1974; Serkowski et al. 1975; Whittet 1977, 1979; Guetter 1977 and others) and on the analysis of color excess ratios of seven O-B2 stars done in Paper II.

The present investigation was undertaken in order to verify these preliminary assumptions about the interstellar extinction law in the area. For this aim we have used multicolor photometry data in the optical, ultraviolet and infrared spectral ranges. The investigation uses stars of $\mathrm{O}$ and B0-B5 spectral classes in the area limited by the coordinates (2000.0 epoch): RA between $2^{\mathrm{h}} 59^{\mathrm{m}}$ and $4^{\mathrm{h}} 08^{\mathrm{m}}$, DEC between $+50^{\circ}$ and $+61^{\circ}$. Many of them belong to the Cam OB1 association. Hereafter, this area will be called the Cam/Per area. Additionally, in some cases we have used 39 stars from the lower right ascensions, between $2^{\mathrm{h}} 30^{\mathrm{m}}$ and $2^{\mathrm{h}} 59^{\mathrm{m}}$, in the same range of declinations. This area contains stars which belong mostly to the Cas OB6 and Per OB1 associations, located in the Perseus spiral arm. Hereafter, it will be called the Cas OB6 area. The stars later than B5 have not been used for the investigation of the reddening law in the optical and ultraviolet ranges, since their intrinsic color indices vary too fast with the spectral class; consequently, the errors in spectral classes ( \pm 1 subclass) lead to an unacceptably low accuracy of the resulting ratios of color excesses defining the interstellar reddening law.

\section{Reddening law in the optical range}

For the investigation of the reddening law in the optical range $(300-700 \mathrm{~nm})$ we have used observations in the Vilnius photometric system of 58 O-B5 type stars with known MK spectral types. The majority of them are from Paper III, and some stars are from Papers I and II. Their spectral types in the MK system are mostly from Hiltner (1956), some are collected from the ADS database. In the area of Paper I additional 14 B-type stars were classified in the MK system by one of us (C. J. Corbally), using the grating spectra with $2.8 \AA$ resolution obtained with the Boller and Chivens spectrograph on the $2.3 \mathrm{~m}$ telescope of Steward Observatory at Kitt Peak. Here we have used seven of them that belong to spectral classes B0-B4.

Color excesses $E_{U-V}, E_{P-V}, E_{X-V}, E_{Y-V}, E_{Z-V}$ and $E_{V-S}$ for all stars were calculated by taking their intrinsic color indices from the Straižys (1992) monograph according to their MK spectral types, as described in our earlier paper (Straižys et al. 1999). With these values of color excesses, graphs $E_{m-V}$ vs. $E_{Y-V}$ are plotted in the upper panels of Figs. 1-5. The " $\times$ " signs are stars of the Cam/Per area, and the open circles are

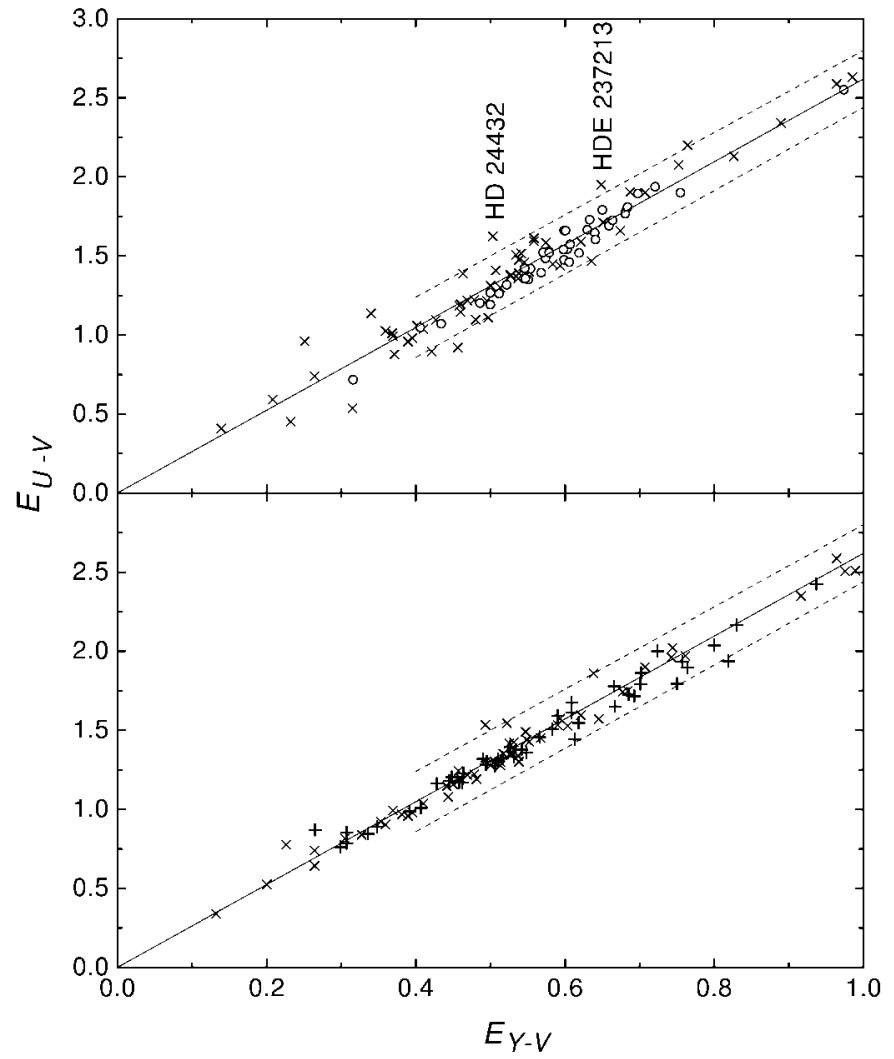

Fig. 1. The dependence of color excesses $E_{U-V}$ and $E_{Y-V}$. The upper panel: color excesses are calculated accepting intrinsic color indices for MK spectral types; the lower panel is the same but accepting intrinsic color indices for photometric spectral types.

stars from the Cas OB6 area observed in Paper III as well as by Sūdžius \& Bobinas (1992).

The solid line on each graph corresponds to the ratio of color excesses for the normal interstellar reddening law (Table 64 from Straižys 1992).

The lower panels of Figs. 1-5 are completely analogous to the upper panels, but here for the calculation of color excesses we used the spectral types (spectral class and luminosity class) determined in Papers I-III from photometric $Q$-parameters. On these graphs, 105 stars of the Cam/Per area are plotted. The "+" signs are the stars for which only photometric spectral classification is available (47 stars), and " $\times$ " signs are the stars with the MK classification (58 stars).

The broken lines on both panels of Fig. 1 show the expected errors of color excesses if the spectral class is wrong by \pm 1 subclass (for example, if a B1 V star is considered as a B0 V or B2 V star). Such errors are appropriate to MK classification's precision.

A glance at the upper panels of Figs. 1-5 shows that, in general, the majority of the stars both in the Cam/Per area and in the Cas OB6 area show normal interstellar reddening. Their deflections from the solid line (normal law) are within the expected errors of spectral classification. However, in Fig. 1 $\left(E_{U-V}\right.$ vs. $\left.E_{Y-V}\right)$ there are several stars which deviate more. These stars may be suspected as having anomalous reddening in the ultraviolet. Some of them will be discussed below, when analysing the interstellar reddening law in the ultraviolet. 


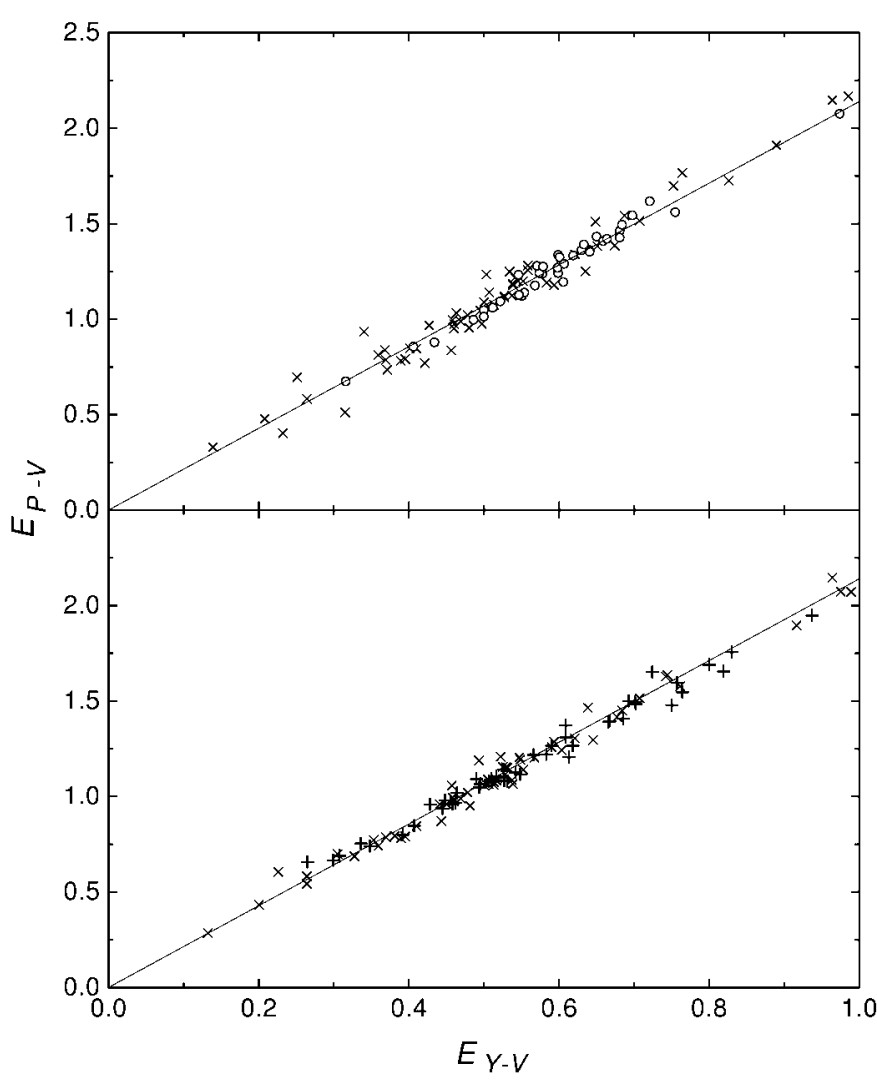

Fig. 2. The dependence of color excesses $E_{P-V}$ and $E_{Y-V}$. Designations are the same as in Fig. 1.

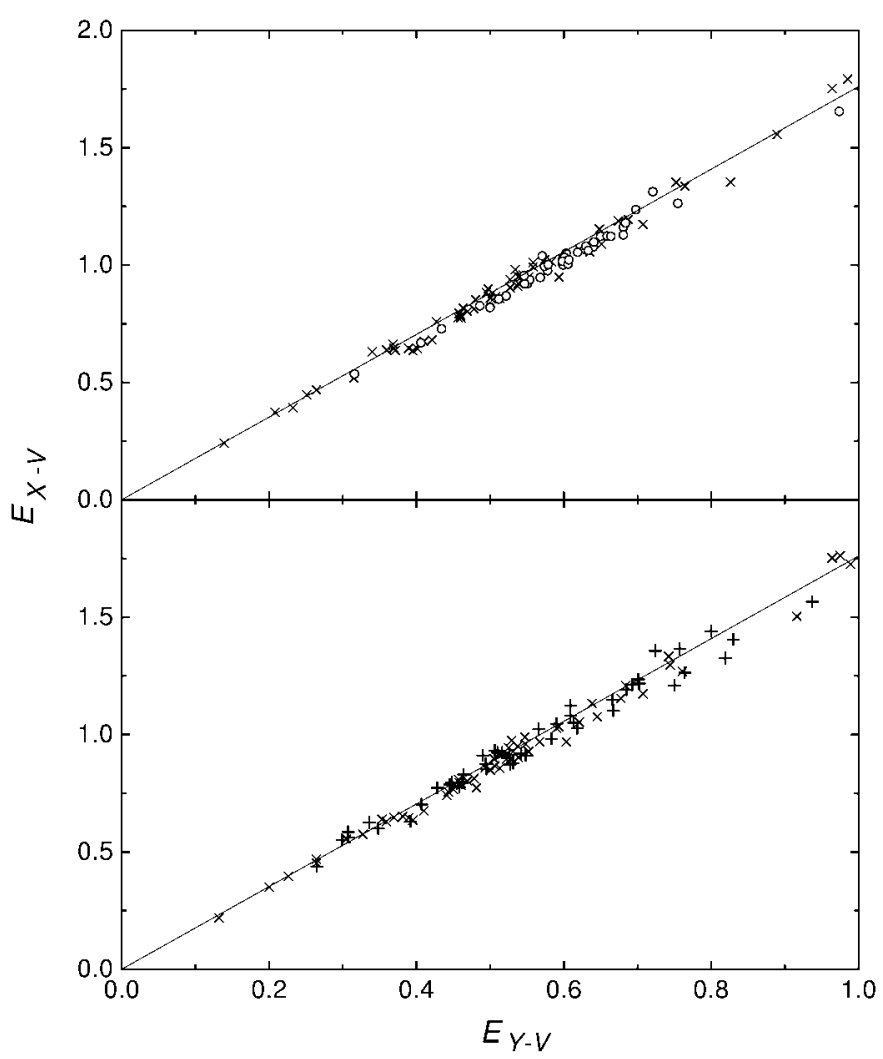

Fig. 3. The dependence of color excesses $E_{X-V}$ and $E_{Y-V}$. Designations are the same as in Fig. 1.

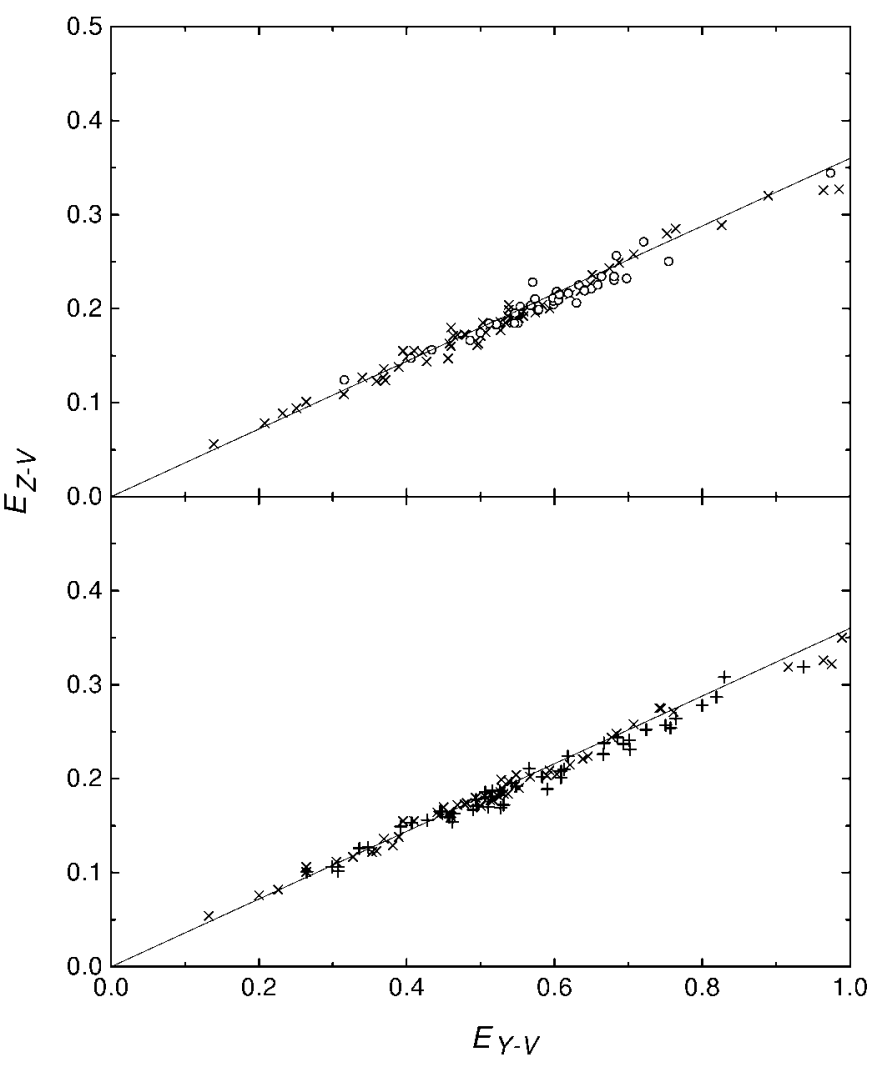

Fig. 4. The dependence of color excesses $E_{Z-V}$ and $E_{Y-V}$. Designations are the same as in Fig. 1.

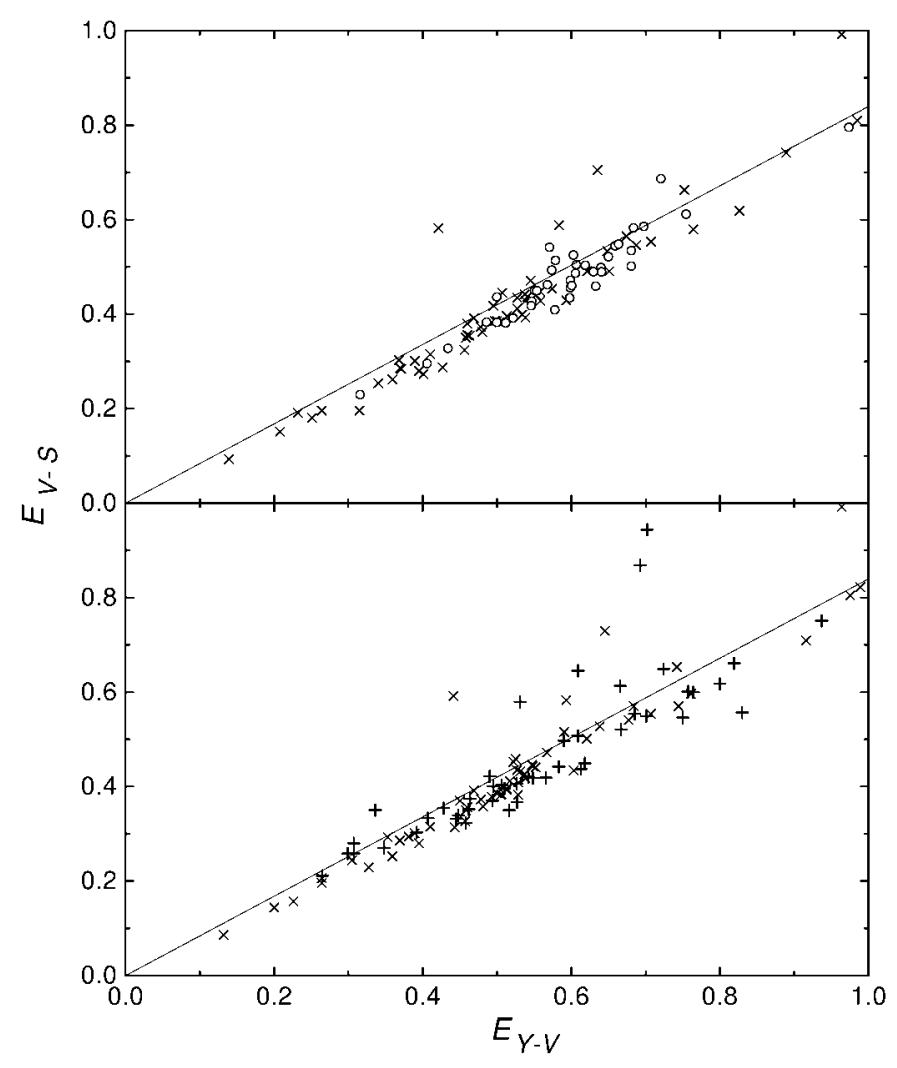

Fig. 5. The dependence of color excesses $E_{V-S}$ and $E_{Y-V}$. Designations are the same as in Fig. 1. 
Another anomaly is observed in Fig. 5 ( $E_{V-S}$ vs. $\left.E_{Y-V}\right)$ where the majority of stars deviate systematically downward by $\sim 0.03$ mag. This means that the extinction in the $S$ passband (situated on $\mathrm{H} \alpha$ line) is somewhat larger than for the normal law. This small systematic effect may be either instrumental or interstellar. A few stars, deviating from the solid line upward, exhibit increased intensity in the $S$ passband. This effect may be caused by an emission component of the $\mathrm{H} \alpha$ line. Most of these stars belong either to Be or related type (HD 19243, HD 21212, HD 22298 and others). They should be excluded from the interstellar law analysis.

The lower panels of Figs. 1 and 2, obtained by using photometric spectral types, include the ultraviolet color indices $U-$ $V$ and $P-V$. They show a smaller scatter of stars around the normal law line than in the corresponding upper panels which use MK spectral types. This means that photometrically determined color excesses are more precise then those determined from MK classification. In Figs. 3-5 the dispersion of stars in both panels is about the same, which reflects the fact that the intrinsic $X-V, Y-V, Z-V$ and $V-S$ color indices vary much less with the spectral class than the ultraviolet indices $U-V$ and $P-$ $V$. Therefore, their color excesses are less affected by the errors of spectral classes. Lower panels of Figs. 1-5 also confirm that the interstellar reddening law in the area is normal and uniform. If the law were variable, then we should expect a considerable scatter of stars around the mean line, increasing with color excess.

The general conclusion is that in the optical spectral range the majority of stars in the Cam/Per and Cas OB6 areas follow the normal interstellar reddening law with some small tendency to exhibit somewhat larger extinction in the red part of the spectrum. Also, some stars exhibit stronger extinction in the ultraviolet at $345 \mathrm{~nm}$.

\section{Reddening law in the ultraviolet}

Many of the OB stars in the Cam/Per area were observed photometrically by the ANS orbiting observatory in the passbands of medium width at 155, 180, 220, 250 and $330 \mathrm{~nm}$. The results of all ANS observations of point sources are published by Wesselius et al. (1982). The system has been used to investigate the interstellar extinction law in different Galactic longitudes and areas by many authors listed in Table 4 of the Straižys (1992) monograph. Meyer \& Savage (1981) have determined the ANS color excess ratios $E_{m-V} / E_{B-V}$ for 1367 stars. They have identified 58 stars and 8 localized regions with the peculiar extinction law. One of their peculiar areas, named R7, overlaps partly our Cam/Per area. The majority of stars in this area were found to exhibit a slightly higher ultraviolet extinction than the mean extinction law. The largest peculiarity in our area is found for the stars HD 24432 (B3 II) and HDE 237213 (B3 Ia). Both these stars show the largest deflection upward in our $E_{U-V}$ vs. $E_{Y-V}$ diagram (Fig. 1). The anomalous extinction in the ultraviolet for HD 24432 was confirmed by Massa et al. (1983) from IUE spectra. The stars in the R7 area also exhibit the increased extinction bump at $220 \mathrm{~nm}$.

Savage et al. (1985) have published a catalog of ultraviolet color excesses in the ANS system and investigated the extinction curves for $1415 \mathrm{O}-\mathrm{B} 7$ stars. They conclude that about $43 \%$ of the stars are affected by peculiar extinction. From their catalog we selected the stars of spectral classes O-B5 in the area limited by the 2000.0 coordinates: RA from $2^{\mathrm{h}} 28^{\mathrm{m}}$ to $4^{\mathrm{h}} 04^{\mathrm{m}}$ and DEC from $+49^{\circ}$ to $+63^{\circ}$. After exclusion of binaries, the number of the stars used is 31 . These stars are plotted in the $E_{m-V}$ vs. $E_{B-V}$ diagrams in Figs. 6a-6e. It is evident that part of the most reddened stars shows a tendency of a larger than normal extinction almost in all ANS photometric passbands. However, for the passband at $330 \mathrm{~nm}$ (panel 6e) the tendency is only marginal.

Later on, Papaj \& Krelowski (1992) published a new catalog of the ANS color excesses for 423 O-B9 stars, using the revised intrinsic color indices. Their results for O-B5 stars in the same area are similar to those of Savage et al. (1985). Only in the $E_{33-V}$ vs. $E_{B-V}$ graph the systematic deviations from the normal law are almost absent.

Several investigators have suspected that the ultraviolet extinction is larger for the stars located in the Perseus spiral arm. The best investigated objects of the Perseus arm are the association Per OB 1 around the double cluster $\mathrm{h}+\chi$ Per (Morgan et al. 1982; Franco et al. 1985; Krelowski \& Strobel 1987) and the association Cas OB6 (Hanson \& Clayton 1993). For the Per OB1 a slightly larger ultraviolet extinction is found, while in the Cas OB6 area the reddening law is found to be normal.

The general conclusion is that the reddening law in the $\mathrm{Cam} /$ Per area in the 155, 180, 220 and $250 \mathrm{~nm}$ passbands shows somewhat larger extinction than on average. In the Cas OB6 area, the law is close to normal, typical for the diffuse dust. In the $330 \mathrm{~nm}$ ANS passband the difference from the mean law is almost absent in both areas.

\section{Reddening law in the infrared}

24 O-A5 stars with $E_{B-V}>0.4$ were found in the Cam/Per area, for which the $K$ magnitudes or $V-K$ color indices were available (here $K$ is the magnitude with the mean wavelength at $2.2 \mu \mathrm{m})$. They were taken either from the 2MASS survey (available at ADS as The 2MASS Database, 2000) or from Castor \& Simon (1983). For each star the ratios $R=$ $A_{V} / E_{B-V}=1.1 E_{V-K} / E_{B-V}$ were calculated. The observed $V$ magnitudes and $B-V$ color indices were taken from Nicolet (1978). MK spectral types were taken mostly from Hiltner (1956). Some spectral types are from other sources. Intrinsic color indices $(B-V)_{0}$ and $(V-K)_{0}$ were taken from Straižys (1992). The $R$ values are found to be between 2.6 and 3.1, with the mean value $R=2.88 \pm 0.12$ which is slightly smaller than the normal value for early-type stars in the diffuse interstellar dust ( $R=3.15$, Straižys 1992). The smaller $R$ ratio is confirmed also by nine O-B stars in the area with their $E_{V-K} / E_{B-V}$ ratios given by Wegner (1993). Their mean $R$ value is 2.95 .

According to Cardelli et al. (1988, 1989), a good correlation exists between the ratio $R$ and the ultraviolet extinction level, when $A_{\lambda}$ are normalized to $A_{V}$ : for large values of $R$ the low ultraviolet extinction $A_{\lambda}$ is observed and vice versa. A somewhat reduced $R$-value, which we find in the Cam/Per 


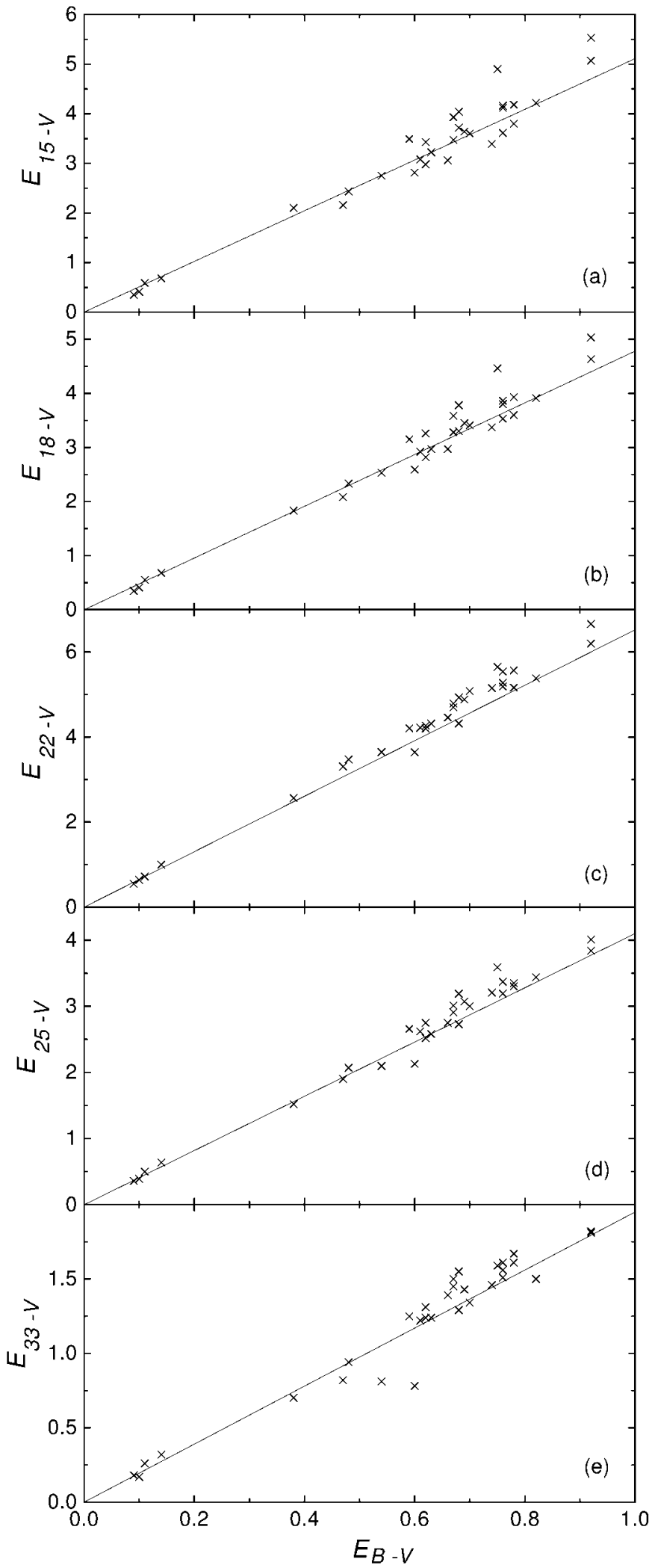

Fig. 6. The dependence of the ultraviolet color excesses in the ANS photometric system on the color excess $E_{B-V}$ of the $U B V$ system.

area, is in perfect agreement with slightly larger interstellar extinction found in the wavelengths shorter than $330 \mathrm{~nm}$.

\section{Wavelength dependence of polarization}

One more effect, reflecting the size distribution of interstellar dust grains, is the wavelength dependence of interstellar polarization. According to Serkowski et al. (1975), $R=$ $5.5 \lambda_{\max }$, while Whittet \& van Breda (1978) find the coefficient 5.6. From the Coyne et al. (1974) catalog of $\lambda_{\max }$ we selected 20 stars within the Galactic longitudes $140-150^{\circ}$. Their mean $\lambda_{\max }=(0.51 \pm 0.03) \mu \mathrm{m}$ gives $R=2.81 \pm 0.15$ for the Serkowski et al. coefficient and $R=2.86 \pm 0.15$ for the Whittet \& van Breda coefficient. These values are in close agreement with the mean $R$ value obtained in the previous section from infrared photometry.

\section{Conclusions}

We have used different methods to estimate the character of the interstellar extinction law in the Milky Way near the border of Camelopardalis, Perseus and Cassiopeia constellations. The results show that the law in the infrared and optical spectral ranges is close to the normal law given in the monograph of one of the authors (Straižys 1992). However, a slightly reduced mean ratio, $R=A_{V} / E_{B-V}=2.9$, is found for O-B type stars in the area. The normal ratio $R=A_{V} / E_{B-V}=3.15$ corresponds to $R_{\mathrm{Vil}}=A_{V} / E_{Y-V}=4.16$ in the Vilnius system. In the case of $R=2.9$, the $R_{\mathrm{Vil}}=3.83$. This value is recommended to use in the future studies of interstellar extinction in the Cam/Per area.

A small deviation from the normal law is also evident in the ANS $330 \mathrm{~nm}$ passband, and at shorter wavelengths the stars are affected by somewhat larger extinction than on average. The same type of deviations is observed in the extinction curves of the Perseus and Camelopardalis stars in the Wegner (2002) atlas. This is in good agreement with the predictions of Cardelli et al. $(1988,1989)$, explaining the variety of interstellar extinction curves found in the ultraviolet.

However, in the optical and near UV range the deviations from the normal law are small, and their influence on the ratios of color excesses of the Vilnius system may be neglected. Consequently, the results of photometric classification of stars in Papers I, II and III do not need to be revised. However, a small difference in the ratio $R$ used in Papers I and II and that found in the present paper needs to be taken into account. The stars having $E_{Y-V}=0.5$ after using a smaller $R$ value will become 1.08 times farther away, while those with $E_{Y-V}=1.0$ will become 1.16 times farther.

Some stars in the area, for example, HD 24432 (B3 II), exhibit a stronger anomaly which begins already at $345 \mathrm{~nm}$, the mean wavelength of the Vilnius $U$ passband. However, there is a possibility that this anomalous extinction law is of circumstellar rather than of interstellar nature.

Acknowledgements. We acknowledge the use of the ADS-database of NASA and the SIMBAD database and the VizieR service of the Centre de Données Astronomiques de Strasbourg. We are also grateful to R. J. Dodd for his report and valuable suggestions. 


\section{References}

Cardelli, J. A., Clayton, G. C., \& Mathis, J. S. 1988, ApJ, 329, L33 Cardelli, J. A., Clayton, G. C., \& Mathis, J. S. 1989, ApJ, 345, 245 Castor, J. I., \& Simon, T. 1983, ApJ, 265, 304

Coyne, G. V., Gehrels, T., \& Serkowski, K. 1974, AJ, 79, 581

Dodd, R. J. 1976, Ap\&SS, 44, 85

Franco, M. L., Magazzu, A., \& Stalio, R. 1985, A\&A, 147, 191

Georgelin, Y. M., \& Georgelin, Y. P. 1976, A\&A, 49, 57

Guetter, H. H. 1977, AJ, 82, 598

Hanson, M. M., \& Clayton, G. C. 1993, AJ, 106, 1947

Hiltner, W. A. 1956, ApJS, 2, 24, 389

Krelowski, J., \& Strobel, A. 1987, A\&A, 175, 186

Massa, D., Savage, B. D., \& Fitzpatrick, E. L. 1983, ApJ, 266, 662

Meyer, D. M., \& Savage, B. D. 1981, ApJ, 248, 545

Morgan, D. H., McLachlan, A., \& Nandy, K. 1982, MNRAS, 198, 779

Nicolet, B. 1978, A\&AS, 34, 1

Papaj, J., \& Krelowski, J. 1992, Acta Astron., 42, 211

Savage, B. D., Massa, D., Meade, M., \& Wesselius, P. R. 1985, ApJS, 59,397

Serkowski, K., Mathewson, D. S., \& Ford, V. L. 1975, ApJ, 196, 261
Serkowski, K., \& Robertson, J. W. 1969, ApJ, 158, 441

Straižys, V. 1992, Multicolor Stellar Photometry (Pachart Publishing House, Tucson, Arizona)

Straižys, V., Corbally, C. J., \& Laugalys, V. 1999, Baltic Astron., 8, 355

Sūdžius, J. 1974, Bull. Vilnius Obs., 39, 18

Sūdžius, J., \& Bobinas, V. 1992, Bull. Vilnius Obs., 86, 59

Taylor, J. H., \& Cordes, J. M. 1993, ApJ, 411, 674

Wegner, W. 1993, Acta Astron., 43, 209

Wegner, W. 2002, Baltic Astron., 11, 1

Wesselius, P. R., van Duinen, R. J., de Jonge, A. R. W., et al. 1982, A\&AS, 49, 427

Whittet, D. C. B. 1977, MNRAS, 180, 29

Whittet, D. C. B. 1979, A\&A, 72, 370

Whittet, D. C. B., \& van Breda, I. C. 1978, A\&A, 66, 57

Zdanavičius, J., Černis, K., Zdanavičius, K., \& Straižys, V. 2001, Baltic Astron., 10, 349 (Paper II)

Zdanavičius, J., \& Zdanavičius, K. 2002, Baltic Astron., 11, 75 (Paper III)

Zdanavičius, K., Zdanavičius, J., \& Kazlauskas, A. 1996, Baltic Astron., 5, 563 (Paper I) 